\title{
Letalidad peatonal en Ciudad de México: análisis general desde una perspectiva de racionalidad limitada
}

Ramiro Flores-Xolocotzi, Investigador en Estudios Urbanos, Unión de Investigadores para la Sustentabilidad, S.A.S. Email: pinos42@hotmail.com. ORCID_ID: 0000-0001-9979-1057

Hatso Hnini Revista de Investigación de Paisajes y Espacio Construido, Vol.1 Núm.1 Año 2021

\author{
Fechas del Artículo: \\ Recibido: 25 agosto 2020 \\ Dictamen: 7 abril 2021 \\ Aceptado: 21 mayo 2021 \\ Publicado: 10 septiembre 2021
}

Forma de citar este artículo: Flores-Xolocotzi, R. (2021).

Letalidad peatonal en Ciudad de México: análisis general desde una perspectiva de racionalidad limitada, Hatso

Hnini Revista de Investigación de Paisajes y Espacio

Construido, Vol. 1 Num.1 pp. 51-67

DOI https://doi.org/10.47386/vol1num1art5_21

\section{Resumen}

Bajo una perspectiva teórica de racionalidad limitada de peatones y de conductores de vehículos, se realizó un estudio sobre tasa de letalidad por atropellamiento peatonal en Ciudad de México considerando causas atribuibles, lugar (intersecciones y no intersecciones de vialidades), horario, día, mes, alcaldía y año del accidente de 1997 a 2017. Para profundizar en el análisis, se estimó un Modelo Poisson Generalizado para analizar estadísticamente el riesgo relativo de muerte por atropellamiento. Se encontraron tres resultados destacables: a) Una mayor letalidad y riesgo relativo de causas atribuibles ajenas a los agentes en fallecimientos peatonales. b) La letalidad es mayor en no intersecciones y el riesgo relativo de fallecimientos en intersecciones es menor en términos porcentuales con respecto a las no intersecciones. c) La letalidad y el riesgo relativo de mortalidad son mayores en causas atribuibles al peatón que en causas atribuibles al conductor. Estos resultados pueden constituir parte de la incertidumbre y restricciones de información que describe la racionalidad limitada. Se concluye que el cruce de peatones en no intersecciones puede provocar mayor letalidad y riesgo relativo de muerte; además de que causas ajenas a la acción de los agentes (peatones y conductores) provocan mayor número de peatones fallecidos.

Palabras clave: Calles, espacio público, letalidad, movilidad, urbanismo.

\section{Abstract}

Considering a theoretical perspective of bounded rationality of drivers and pedestrians in pedestrian collisions, a research was carried out about pedestrian lethality rate in Mexico City considering attributable causes to persons or other causes, place (intersections and non-intersections of roads), time of the day, day, month, local municipalities and year of the accident from 1997 to 2017. To the analysis was estimated a Generalized Poisson Model. With the model results, the relative risk of pedestrian death after being run over was analyzed. There are three remarkable results: Lethality and relative risk of deaths increased due to non-attributable causes of the agents. Secondly, that lethality is higher in non-intersections and that the relative risk of deaths in intersections is lower in percentage terms concerning non-intersections. Third, that lethality and the relative risk of deaths are greater in attributable causes of pedestrians than in attributable causes of drivers. These results may constitute part of the uncertainty and information constraints that describe bounded rationality. The conclusion is that crossing pedestrians at non-interference crossings can increase lethality and increase death's relative risk. Also, it was observed that causes unrelated to agent's actions (pedestrians and drivers) cause more pedestrian deaths.

Keywords: Streets, public space, lethality, mobility, urbanism.

\section{Introducción}

Los modelos sobre movilidad describen entre otros aspectos, la interacción entre personas que hacen uso de vías de transporte (peatones y conductores) además de que pueden evidenciar problemas urbanos como los accidentes en dichas vías (Guimarães y da Silva, 2019; Lukusa y Kin, 2020; Másilková, 2017; Värnild et al., 2020). En este estudio se referirá a dichas personas como agentes tomadores de decisión tal como lo hacen Plata et al. (2009) y Plata (2013) en su análisis sobre racionalidad limitada. 
Letalidad peatonal en Ciudad de México: análisis general desde una perspectiva de racionalidad limitada

Ramiro Flores-Xolocotzi

Hatso Hnini Revista de Investigación de Paisajes y Espacio Construido, Vol.1 Núm.1 Año 2021

De los tipos de accidentes en donde están involucrados vehículos, peatones, ciclistas, motociclistas entre otros usuarios de calles y avenidas es de interés para esta investigación la colisión peatonal (en adelante $\mathrm{CP}$ ) o atropellamiento de peatones en donde están involucrados conductores de los diferentes tipos de vehículos de transporte involucrados (Bambarén y Chú, 2013; Domingo, 2018; Flórez et al., 2018; Ruiz y Herrera, 2016).

De acuerdo con el Instituto Nacional de Estadística y Geografía (INEGI, 2009), en México, dichos vehículos pueden ser automóviles, camionetas para pasajeros, microbuses, camión urbano de pasajeros, ómnibuses, tranvías, camionetas de carga, camión de carga, tractores con o sin remolque, ferrocarril, motocicleta, bicicletas u otros vehículos. La CP es un subtipo de accidentes vehiculares que constituyen un problema de salud pública, cuyos datos de mortalidad para el año 2016 implican que los peatones son quienes tienen el mayor porcentaje de fallecimiento $(7,137$ atropellamientos fatales que son un $44 \%$ aproximado de los fallecimientos por accidentes de tránsito) (Secretaría de Salud, 2018).

No obstante, la CP en ciudades, puede abordarse también como un problema conductual de toma de decisiones de los agentes involucrados: conductor del vehículo y peatón. Tales decisiones podrían determinar en mayor o menor medida la letalidad de la colisión y asimismo conocer la responsabilidad del conductor y peatón en la CP (Bjørnskau, 2017; Chen et al., 2016; Chen et al., 2019; Elvik, 2014; Fuller, 2005; Mukherjee y Mitra, 2019).

\section{Marco Teórico}

La economía conductual aplicada a accidentes de tránsito permite estudiar a la CP como un escenario que pueda tomar en cuenta los diversos contextos en que pueden ocurrir, considerando que conductores y peatones toman decisiones bajo incertidumbre (Shaoman y Ying, 2015; Tang et al., 2015). Lo anterior permite analizar la CP bajo un marco teórico de racionalidad limitada (limitaciones cognitivas de los agentes), tal como lo han hecho Shaoman y Ying (2015), Schmidt-Daffy (2014) y Tang et al. (2015). De acuerdo con Sivak (2002); Elvik (2015); Elvik (2016) y Schmidt-Daffy (2014) el modelo de racionalidad limitada (en adelante RL) es útil en la comprensión y análisis de los accidentes de tráfico, ya que ayudan a comprender las conductas de peatones y conductores bajo el enfoque de que es generalmente adaptativa.
Siguiendo a Sivak (2002) y Shinar (2017) para el caso del peatón y del conductor de un vehículo, ambos agentes están sujetos a diversos contextos con posibles restricciones de tiempo y de información de lo que puede hacer el otro, lo cual provoca situaciones inseguras. De esta forma el modelo teórico de RL aplicada a CP puede considerarse como un proceso no optimizador y de conducta adaptativa de agentes reales que descansa en un procesamiento de recur-sos limitados (Shinar, 2017). Por el contrario, consi-derando a Shinar (2017), un modelo de racionalidad ilimitado (característico del modelo de racionalidad sustantiva o estándar sin restricciones de informa-ción) consideraría todas las alternativas, junto con el uso de la información necesaria para que cada to-mador de decisiones en el sistema vial, haga una elección.

Bajo un contexto ideal del modelo de racionalidad sustantiva, el peatón tendría que considerar toda la información necesaria para tomar la decisión de cruzar 0 no cruzar la calle sea en "intersección" (esquinas) o en no "intersección", por ejemplo: señalizaciones, velocidad y tipo de vehículo; intención, estado mental y condiciones físicas de los conductores (las cuales el otro agente desconoce) (Sivak, 2002; Sent, 2005; Jiménez, 2010; Schilirò, 2018).

Considerando lo anterior, este trabajo opta por considerar un análisis de letalidad y mortalidad de la CP siguiendo el marco teórico de RL de acuerdo con Simon (2019). Por lo cual, se parte del supuesto que en caso de CP, es el peatón atropellado, el que está más expuesto a ser herido y en una situación peor: morir al ser arrollado o empujado por un vehículo de transporte. Se destaca que la base de datos empleada en este estudio considerará colisiones peatonales en las cuales se analizará estadísticamente sólo letalidad y riesgo relativo de muerte peatonal (incrementos y decrementos en la tasa de peatones atropellados fallecidos).

Siguiendo a Villa et al. (2014) se entendería letalidad como el número de peatones atropellados fallecidos en la CP con respecto al total de atropellados por colisión. Así esta investigación, tiene los siguientes objetivos:

1.- Describir la letalidad peatonal por atropellamiento en zonas urbanas de Ciudad de México (en adelante CdMx) considerando la causa atribuible (acción del peatón, acción del conductor, otra causa no atribuible a la acción del conductor o peatón), 
Letalidad peatonal en Ciudad de México: análisis general desde una perspectiva de racionalidad limitada

Ramiro Flores-Xolocotzi

Hatso Hnini Revista de Investigación de Paisajes y Espacio Construido, Vol.1 Núm.1 Año 2021

lugar del accidente (en la intersección o esquina de la vialidad y en la no intersección de la vialidad). Además se considerarán algunas variables temporales en las que ocurrió el atropellamiento: horario (madrugada, mañana, tarde y noche); día de la semana y mes del año en un horizonte temporal anual de 1997 a 2017.

2.- Estimar un modelo de conteo que permita analizar el número de peatones fallecidos por $\mathrm{CP}$ además de estimar el riesgo relativo (en adelante $R R$ ) de fallecimiento en función de la acción del peatón, la acción del conductor o causa no atribuible a ambos, junto con otras variables independientes como: lugar de atropellamiento ("en intersección" o "en no intersección"), horario, día, mes, alcaldía, año del accidente (1997 a 2017). En las estimaciones se empleará también una variable independiente de tipo offset (o de exposición) constituida por el logaritmo natural del número total de peatones atropellados por colisión.

3.-Analizar los resultados obtenidos bajo un enfoque conductual de RL de los agentes involucrados considerando letalidad y $R R$ de fallecimiento por atropellamiento.

\section{Metodología}

Es necesario señalar las limitantes de esta investigación, primero se tiene que considerar que los modelos Poisson estimados y analizados en este trabajo, son de tipo descriptivo más no predictivo. En cuanto a decisiones del agente, sólo considera variables relacionadas con la causa atribuible y la posible elección del lugar de cruce peatonal de la vialidad expresada a través del lugar del accidente (en intersección o en no intersección).

Por lo anterior, el modelo y los resultados no consideran diversos aspectos personales relacionados con el contexto o los motivos particulares de la elección, por ejemplo: edad y estado físico de los agentes; habilidades de manejo; discapacidades físicas y mentales; consumo de alcohol y drogas; variables tiempo/distancia para llegar a destino; elementos distractorios (uso de celular, audífonos); diseño urbano (pavimento, alumbrado público, puentes peatonales, pasos de cebra); factores climáticos; velocidad del vehículo, otros (Dong et al., 2014; Eichelberger et al., 2018; Quistberg et al., 2015; Shinar, 2017; Thompson et al., 2012).
Si bien las investigaciones sobre atropellamientos peatonales pueden ser abordadas a través de otros modelos de tipo sociodemográfico y de salud pública (modelos epidemiológicos) que describan la colisión peatonal, tales enfoques no son abordados en este trabajo. Finalmente se hace la aclaración, que esta investigación tampoco comprende un análisis de frecuencia de colisiones peatonales como tal y sus causas. Ni tampoco realiza un análisis sobre la fatalidad del accidente peatonal en el sentido de si hubo o no pérdidas de vidas humanas tal como lo describe INEGI (2016). Tanto la frecuencia de colisiones, como la fatalidad del accidente y sus respectivas múltiples causas, son otro enfoque de investigación, que se realizó de manera paralela para otro estudio de índole social.

La investigación que se realizó fue de tipo cuantitativa, descriptiva y ex post facto. Para ello se elaboró en Excel, versión 365 (Microsoft Excel, 2021) una base de datos con información y estadísticas de atropellamientos peatonales ocurridas en zonas urbanas (en adelante ZU) de CdMx de 1997 a 2017 y que reporta INEGI (2020) para dichos años. El número de observaciones de la base de datos fue de 22,735 colisiones peatonales registradas en $\mathrm{ZU}$ de CdMx. De acuerdo con INEGI (2009), se entiende por ZU aquellas áreas habitadas con continuidad física en todas direcciones hasta ser interrumpida, notoriamente por terrenos de uso no urbano como bosques, campos de siembra y/o cuerpos de agua. Estas zonas tienen asentamientos humanos concentrados de más de 15,000 habitantes y cuentan con infraestructura, equipamiento y servicios urbanos.

Las variables que constituyeron la base de datos se encuentran mencionadas en Tabla 1. Posteriormente se elaboraron tablas con estadísticas descriptivas de porcentaje de ocurrencia de letalidad por atropellamiento peatonal por año, lugar del accidente (en intersección y en no intersección), causa del accidente (atribuible al peatón, atribuible al conductor, otra), horario del accidente (madrugada, mañana, tarde, noche), día del accidente, mes, año y alcaldía de CdMx. 
Letalidad peatonal en Ciudad de México: análisis general desde una perspectiva de racionalidad limitada

Ramiro Flores-Xolocotzi

Hatso Hnini Revista de Investigación de Paisajes y Espacio Construido, Vol.1 Núm.1 Año 2021

Tabla 1. Variables que se consideraron en el análisis estadístico (obtenidas de colisiones peatonales registradas en CdMx de 1997 a 2017)

\begin{tabular}{|c|c|}
\hline Variable & Tipo de variable \\
\hline $\begin{array}{l}\text { Yi= Número de peatones atropellados fallecidos } \\
\text { (variable dependiente) }\end{array}$ & Cuantitativa (conteo) \\
\hline Lugar del accidente & 0 = No Intersección y 1 = Intersección \\
\hline Causa atribuible a peatón & BASE $0=$ No y $1=\mathrm{Si}$ \\
\hline Causa atribuible a conductor & $0=$ No y $1=\mathrm{Si}$ \\
\hline $\begin{array}{l}\text { Causa no atribuible a conductor ni a peatón (falla } \\
\text { mecánica de vehículo, condición de vialidad u otra } \\
\text { no especificada) }\end{array}$ & $0=$ No y $1=\mathrm{Si}$ \\
\hline Horario del accidente: Madrugada & BASE $0=$ No y $1=\mathrm{Si}$ \\
\hline Mañana & $0=$ No $y=S i$ \\
\hline Tarde & $0=$ No y $1=\mathrm{Si}$ \\
\hline Noche & $0=$ No y $1=S i$ \\
\hline Día del accidente: Lunes & BASE $0=$ No y $1=\mathrm{Si}$ \\
\hline Martes & $0=$ No y $1=\mathrm{Si}$ \\
\hline Miércoles & $0=$ No y $1=\mathrm{Si}$ \\
\hline Jueves & $0=$ No y $1=\mathrm{Si}$ \\
\hline Viernes & $0=$ No y $1=\mathrm{Si}$ \\
\hline Sábado & $0=$ No y $1=\mathrm{Si}$ \\
\hline Domingo & $0=$ No y $1=\mathrm{Si}$ \\
\hline Alcaldía: Álvaro Obregón & BASE $0=$ No y $1=\mathrm{Si}$ \\
\hline Azcapotzalco & $0=$ No y $1=S i$ \\
\hline Benito Juárez & $0=$ No y $1=\mathrm{Si}$ \\
\hline Coyoacán & $0=$ No y $1=\mathrm{Si}$ \\
\hline Cuajimalpa & $0=$ No y $1=\mathrm{Si}$ \\
\hline Cuauhtémoc & $0=$ No y $1=\mathrm{Si}$ \\
\hline Gustavo A. Madero & $0=$ No y $1=\mathrm{Si}$ \\
\hline Iztacalco & $0=$ No y $1=S i$ \\
\hline Iztapalapa & $0=$ No y $1=\mathrm{Si}$ \\
\hline Magdalena Contreras & $0=$ No y $1=\mathrm{Si}$ \\
\hline Miguel Hidalgo & $0=$ No y $1=\mathrm{Si}$ \\
\hline Milpa Alta & $0=$ No y $1=\mathrm{Si}$ \\
\hline Tláhuac & $0=$ No y $1=\mathrm{Si}$ \\
\hline Tlalpan & $0=$ No y $1=\mathrm{Si}$ \\
\hline
\end{tabular}


Letalidad peatonal en Ciudad de México: análisis general desde una perspectiva de racionalidad limitada

Ramiro Flores-Xolocotzi

Hatso Hnini Revista de Investigación de Paisajes y Espacio Construido, Vol.1 Núm.1 Año 2021

\begin{tabular}{|l|l|}
\hline Venustiano Carranza & $0=$ No y $1=\mathrm{Si}$ \\
\hline Xochimilco & $0=$ No y $1=\mathrm{Si}$ \\
\hline Enero & BASE $0=\mathrm{No}$ y $1=\mathrm{Si}$ \\
\hline Febrero & $0=$ No y $1=\mathrm{Si}$ \\
\hline Marzo & $0=$ No y $1=\mathrm{Si}$ \\
\hline Abril & $0=$ No y $1=\mathrm{Si}$ \\
\hline Mayo & $0=$ No y $1=\mathrm{Si}$ \\
\hline Junio & $0=$ No y $1=\mathrm{Si}$ \\
\hline Julio & $0=$ No y $1=\mathrm{Si}$ \\
\hline Agosto & $0=$ No y $1=\mathrm{Si}$ \\
\hline Septiembre & $0=$ No y $1=\mathrm{Si}$ \\
\hline Octubre & $0=$ No y $1=\mathrm{Si}$ \\
\hline Noviembre & $0=$ No y $1=\mathrm{Si}$ \\
\hline Diciembre & $0=$ No y $1=\mathrm{Si}$ \\
\hline Interacción: Martes * Tarde & $0=$ No y $1=\mathrm{Si}$ \\
\hline Sábado * Noche & $0=$ No y $1=\mathrm{Si}$ \\
\hline Año & Variable que expresa tendencia en el tiempo \\
\hline $\begin{array}{l}\text { Variable offset: InPAi (logaritmo natural del número } \\
\text { total de peatones atropellados por colisión) }\end{array}$ & Cuantitativa (parámetro igual a 1) \\
\hline
\end{tabular}

Fuente: Elaboración propia considerando información de INEGI (2020)

\section{Modelos Poisson}

Con la base de datos, se estimó un primer modelo Poisson considerando como variable dependiente a $Y_{i}$ el número de peatones fallecidos por CP. Para analizar la mortalidad se incorporaron como variables independientes: lugar del accidente, causa atribuible, horario del accidente, día del accidente, alcaldía del accidente, mes del accidente, año del accidente (ver Tabla 1). Igualmente, se probaron diferentes interacciones entre las variables independientes mencionadas, permaneciendo en los modelos dos interacciones: martes*tarde y sábado*noche que se seleccionaron por su contribución en la bondad de ajuste de acuerdo a los Criterios de Información de Aikake y Bayesiano (AIC y BIC, respectivamente).

Además, se añadió a los modelos como variable independiente una variable de exposición también denominada como offset y, que estuvo representada por el logaritmo natural del número total de peatones atropellados por CP $\left(\operatorname{InPA} A_{i}\right)$, con un valor de parámetro igual a 1. La variable offset (de exposición) influye directamente sobre la variable dependiente $Y_{i}$, ya que es lógico asumir que a mayor número de peatones atropellados podrá haber mayor número de peatones fallecidos. De esta forma, la variable offset representa el tamaño de la unidad de peatones totales atropellados sobre la cual se realizan los conteos de peatones fallecidos en cada CP (ver Ecuación 6) (Cummings, 2019; Hilbe, 2020). No debe perderse de vista que esta variable offset se obtiene matemáticamente a partir de la razón del $\ln \left(\mathrm{Y}_{\mathrm{i}} / \mathrm{Pa}_{\mathrm{i}}\right)$ y un posterior despeje del $\operatorname{InPA} A_{i}$, aplicando reglas de logaritmos, tal como se observa en las ecuaciones 4, 5 y 6 .

Considerando lo anterior, los modelos finales tuvieron un total de 42 variables independientes. Sobre la variable independiente año, esta se transformó linealmente de acuerdo con lo recomendado por Cummings (2019), para modelos Poisson. La fórmula de transformación fue: [(Año-1997)/19]. 


\section{Ramiro Flores-Xolocotzi}

Hatso Hnini Revista de Investigación de Paisajes y Espacio Construido, Vol.1 Núm.1 Año 2021

La transformación anterior, contribuyó a evitar problemas de colinealidad en la matriz de variables independientes en las regresiones estimadas, como se describe posteriormente en resultados. De esta manera los modelos Poisson estimados en esta investigación permiten analizar la influencia de la variable offset junto con el resto de las variables independientes sobre la variable dependiente.

El primer modelo estimado fue un Modelo de Regresión Poisson simple para datos de conteo en el que las variables independientes y la variable offset son incluidas en el modelo vía una función link invertible que describe la relación del predictor lineal $x_{i} \beta=\eta_{i}$ con el valor esperado de la respuesta que es $\theta_{i}$ De acuerdo con Allison (2012), Harris et al. (2012) y Long y Freese (2014), el modelo Poisson simple tiene la forma:

$$
f\left(y_{i} ; \theta_{i}\right)=\frac{\theta_{i}^{y_{i}} e^{-\theta_{i}}}{y_{i} !}, \quad y_{i}=0,1,2, \ldots, \quad \theta_{i}>0 \ldots(1)
$$

De acuerdo con Harris et al. (2012), el valor esperado en términos de la función log link inversa estaría dado por:

$$
\theta_{i}=\exp \left(x_{i} \beta\right)
$$

En donde $x_{i}$ es un vector de variables independientes y su acompañante $\beta$ es el vector de parámetros estimados en la regresión.

Tomando en cuenta que habrá una variable offset con un parámetro igual a 1 , de acuerdo con Cummings (2019), la ecuación lineal inicial del Modelo de Regresión Poisson estimado tiene la siguiente forma:

1.- Considerando logaritmos a ambos lados de la ecuación:

$$
\begin{gathered}
\ln \left(\frac{Y_{i}}{P A_{i}}\right)=\ln \beta_{0}+\ln \beta_{1} X_{1}+\ln \beta_{2} X_{2}+ \\
\ldots+\ln \beta_{k} X_{k}
\end{gathered}
$$

2.- Considerando que $\ln \beta=b$, la ecuación se puede reescribir como:

$$
\begin{aligned}
\ln \left(\frac{Y_{i}}{P A_{i}}\right)= & b_{0}+b_{1} X_{1}+b_{2} X_{2}+ \\
& \ldots+b_{k} X_{k}
\end{aligned}
$$

3.- Por propiedad del cociente de logaritmos, el lado izquierdo de la ecuación que contiene la razón $\ln \left(\mathrm{Y}_{\mathrm{i}}\right)$ $\left.P A_{i}\right)$, se puede reescribir como:

$$
\begin{gathered}
\ln Y_{i}-\ln P A_{i}=b_{0}+b_{1} X_{1}+b_{2} X_{2}+ \\
\ldots+b_{k} X_{k}
\end{gathered}
$$

4.- Para obtener el modelo final que se estimará en esta investigación y considerando que habrá una variable offset (de exposición), se despejará el término $\operatorname{lnPA} A_{i}$ al lado derecho de la ecuación. Siendo dicho término del lado derecho, la variable offset (logaritmo natural del número total de peatones atropellados por $\mathrm{CP}$ ):

$$
\begin{array}{r}
\ln Y_{i}=b_{0}+b_{1} X_{1}+b_{2} X_{2}+ \\
\ldots+b_{k} X_{k}+\ln P A_{i}
\end{array}
$$

Consecuentemente el modelo Poisson simple en esta investigación tienen la forma descrita en la ecuación 6.

De acuerdo con Allison (2012) en el modelo Poisson para la variable dependiente $Y i$, la relación entre la media y su varianza es: $E(Y)=\operatorname{Var}(Y)$. Para analizar este supuesto y siguiendo a Hilbe (2020) se empleó el Estadístico de Dispersión de Pearson (Pearson/ $\mathrm{GL}$ ). En donde $\mathrm{GL}=$ número de observaciones del modelo menos el número de parámetros estimados.

Considerando lo anterior, las reglas de decisión para el Estadístico de Dispersión de Pearson son:

a) $P / G L>1$ hay sobredispersión, en donde:

$$
\operatorname{Var}(Y)>E(Y) \text {; }
$$

b) $\mathrm{P} / \mathrm{GL}<1$ hay subdispersión, en donde:

$\operatorname{Var}(\mathrm{Y})<\mathrm{E}(\mathrm{Y})$

c) $\mathrm{P} / \mathrm{GL}=1$ hay equidispersion, en donde:

$\operatorname{Var}(Y)=E(Y)$, (entendiendo que en el modelo Poisson: media y varianza son idénticas).

Debido a que se encontró subdispersión en el Modelo de Regresión Poisson ajustado a los datos de esta investigación, lo cual pudiese provocar que los parámetros estimados no fuesen significativos cuando deben de serlo, se procedió a estimar un Modelo de Regresión Poisson Generalizado (en adelante Modelo PG) (Consul y Famoye, 1992; Hilbe, 2020; Chou, 2009; Cui et al., 2006; Famoye et al., 2004). 
Para ello se introduce un parámetro adicional " $\delta$ " de acuerdo con Consul y Famoye (1992), Husain y Hossain (2015), Famoye (1993) y Harris et al. (2012). El modelo tendrá la siguiente forma:

$$
\begin{gathered}
f\left(y_{i}, \theta_{i}, \delta\right)=\frac{\theta_{i}\left(\theta_{i}+\delta y_{i}\right)^{y_{i}-1} e^{-\theta_{i}-\delta y_{i}}}{y_{i} !}, \\
y_{i}=0,1,2, \ldots
\end{gathered}
$$

En donde se consideraría que:

$$
\theta_{i}>0 \quad y \max \left(-1, \frac{\theta_{i}}{4}\right)<\delta<1
$$

En este modelo PG, la media sería:

$$
\mu_{i}=E\left(Y_{i}\right)=\frac{\theta_{i}}{1-\delta}
$$

Además, la varianza quedaría definida como:

$$
\operatorname{Var}\left(Y_{i}\right)=\frac{\theta_{i}}{(1-\delta)^{3}}=\frac{1}{(1-\delta)^{2}} E\left(Y_{i}\right)=\phi E\left(Y_{i}\right) \ldots
$$

En la ecuación 10 de la varianza, el término $\Phi$ sería un factor de dispersión. De modo que considerando a $\delta$ (delta):

a) $\delta=0$ hay equidispersión y por lo tanto el Modelo PG se reduce al modelo Poisson simple con parámetro $\Phi_{i}$ de acuerdo a la ecuación 1.

b) $\delta>0$ hay sobredispersión en los datos.

c) $\delta<0$ hay subdispersión en los datos.

Finalmente, de acuerdo con Consul y Famoye (1992), Famoye (1993) y Harris et al. (2012), la función Log de máxima verosimilitud a estimar dentro del Modelo PG sería:

$$
L=\sum_{i=1}^{n}\left\{\ln \theta_{i}+\left(y_{i}-1\right) \ln \left(\theta_{i}+\delta y_{i}\right)-\left(\theta_{i}+\delta y_{i}\right)-\ln y_{i} !\right\}
$$

En donde las variables independientes se introducen a la regresión a través de la siguiente relación (Consul y Famoye, 1992; Harris et al., 2012):

$$
\ln \frac{\theta_{i}}{1-\delta}=\beta_{0}+\sum_{j=1}^{p} x_{i j} \beta_{j}+\ln P A_{i}
$$

En donde $x_{i j}$ es la i-ésima observación de la j-ésima variable independiente, $\mathrm{p}$ es el número de variables independientes en el modelo y $\beta_{\mathrm{j}}$ son los parámetros estimados en la regresión y $\operatorname{lnP} A_{i}$ es la variable offset (logaritmo natural del número total de peatones atropellados por colisión) (Cummings, 2019). De acuerdo con Harris et al. (2012), esta generalización sobre el Modelo Poisson permite modelar datos de conteo que sean equidispersos, subdispersos y sobredispersos a diferencia del Modelo de Poisson simple (Ecuación 1) que impone una restricción de equidispersion sobre la relación media-varianza de la variable de conteo.

Hay que señalar que la subdispersión en datos de accidentes de tráfico es poco frecuente de encontrar, sin embargo se puede presentar (Lu y Tolliver, 2016). Los modelos de regresión se estimaron en Statistical Analysis System Software, versión 9.4 (SAS Software, 2021) mediante los procedimientos GENMOD (Modelo de Regresión Poisson simple) y NLMIXED (Modelo de Regresión PG) junto con los análisis de colinealidad, de dispersión, medidas de bondad de ajuste y la Prueba de la Razón de Verosimilitud (LRTEST) para contrastar la hipótesis de subdispersión de los datos (Allison, 2012; Chou, 2009; Cummings, 2019; Famoye et al., 2004; Hilbe, 2020; Long y Freese, 2014).

\section{Resultados}

\section{Estadística descriptiva sobre fallecimientos y heridos}

La base de datos comprendió 22,735 colisiones peatonales registradas en ZU de CdMx de 1997 a 2017. El rango de peatones atropellados fallecidos por colisión fluctuó en términos totales entre 0 a 3 fallecidos por colisión (con un $0.0176 \%$ para 3 fallecidos hasta $87.8338 \%$ para cero fallecidos). Mientras que el número de peatones heridos por colisión fluctuó de 0 a 8 por colisión (con un $0.0044 \%$ para 8 heridos hasta $11.6824 \%$ para cero peatones heridos) (ver Tabla 2).

\section{Colisiones peatonales, mortalidad y letalidad}

Con respecto a CP en ZU de CdMx se encontró un valor promedio anual de 1082.619 colisiones peatonales con un máximo de 3211 colisiones peatonales en 1999 y un mínimo de 739 en 2015. 
Tabla 2. Porcentaje de CP considerando número de peatones fallecidos y heridos.

\begin{tabular}{|c|c|c|}
\hline $\begin{array}{c}\text { Número de peatones } \\
\text { afectados por CP }\end{array}$ & $\begin{array}{c}\% \text { de CP con determinado número } \\
\text { de peatones fallecidos }\end{array}$ & $\begin{array}{c}\text { \% de CP con determinado } \\
\text { número de peatones heridos }\end{array}$ \\
\hline 0 & 87.8338 & 11.6824 \\
\hline 1 & 11.9419 & 83.268 \\
\hline 2 & 0.2067 & 4.4777 \\
\hline 3 & 0.0176 & 0.4311 \\
\hline 4 & - & 0.0836 \\
\hline 5 & - & 0.0308 \\
\hline 6 & - & 0.0044 \\
\hline 7 & - & 0.0176 \\
\hline 8 & - & 0.0044 \\
\hline
\end{tabular}

Elaboración propia con datos de INEGI (2020)

De tasas de letalidad en colisiones peatonales anuales por año en ZU de CdMx, se encontró un valor promedio de $12.41 \%$, con un máximo de $14.80 \%$ en 2006 y un mínimo de $3.26 \%$ en 1999 (ver Tabla 3). Se hace la observación que para el año 1999 el valor de $3.26 \%$ representa atípicamente una tasa de letalidad a la baja. Para interpretar este valor se debe tomar en cuenta que en 1999 se presentaron 3211 colisiones peatonales (el mayor valor para el horizonte de tiempo estudiado).

También, se encontró que la variable dependiente "peatones atropellados fallecidos por CP" tiene una media de 0.1241 y una varianza de 0.1139 para el período de estudio (1997 a 2017). El análisis general de esta información llevó a considerar la posibilidad de una subdispersión en los datos en el análisis de regresión Poisson que se analizará más adelante. Respecto al total de "peatones atropellados por CP" se encontró una media de 1.066 y una varianza de 0.092 para el mismo período de estudio.

\section{Letalidad por causa, lugar, horario, día, mes y} alcaldía

Los resultados de letalidad considerando las variables independientes bajo estudio, se encuentran reportados en (ver Tabla 4) en forma de tasas de letalidad. Se observa que la letalidad por horario en promedio durante los años de estudio (1997 a 2017) es mayor durante las madrugadas $(22.19 \%)$, los días domingo (15.88\%), durante el mes de diciembre (14.14\%) y en la alcaldía Xochimilco (20.51\%).

Considerando que el número anual de $\mathrm{CP}$ que le sigue a 3211 es de 1248 en el año 2012, en esas
3211 colisiones hubo el mayor valor de peatones atropellados que fue de 3252 que supera al siguiente valor total de 1338 peatones atropellados en 1997. Sin embargo de 3252 peatones atropellados en 1999, sólo 106 fallecieron (segundo valor más bajo de mortalidad) (ver Tabla 3). Consecuentemente, la interpretación de este resultado, debe hacerse considerando lo anterior.

\section{Análisis de la letalidad}

El análisis y discusión bajo la teoría de la RL tiene que considerar resultados relacionados con acciones o conductas atribuibles a los agentes involucrados. Considerando lo anterior, se observa que la letalidad es mayor cuando la causa se atribuye a causas no atribuibles a la acción del conductor ni del peatón (20.54\%), esas causas de acuerdo con INEGI, (2020) pueden ser debidas a malas condiciones del camino, falla del vehículo o alguna otra causa no atribuible al peatón o conductor (ver Tabla 4).

Siguiendo con el análisis de acciones, la letalidad asociada a causas atribuibles al peatón es mayor $(14.35 \%)$ frente a causas atribuibles al conductor (12.31\%) (ver Tabla 4.). A esto hay que añadir que la letalidad es mayor cuando la CP ocurre en las no intersecciones más que en las intersecciones de las vialidades $(13.19 \%$ y $11.53 \%$ respectivamente) (ver Tabla 4.). Este resultado sobre el lugar de atropellamiento puede proporcionar un indicador de la elección de cruce de la vialidad que toma el peatón y el resultado de la letalidad por la elección de cruzar o no en determinado lugar. 
Tabla 3. Colisiones peatonales, mortalidad y tasa de letalidad de las colisiones peatonales por año de 1997 a 2017 en ZU de CdMx. (En negrillas valor mínimo, valor máximo y media de la tasa de letalidad)

\begin{tabular}{|c|c|c|c|c|}
\hline Año & $\begin{array}{l}\text { Total colisiones } \\
\text { peatonales }\end{array}$ & $\begin{array}{l}\text { Total peatones atro- } \\
\text { pellados fallecidos }\end{array}$ & $\begin{array}{l}\text { Total Peatones } \\
\text { atropellados }\end{array}$ & \begin{tabular}{|c|} 
Tasa de letalidad porcentual \\
(fallecidos entre total de \\
atropellados por 100)
\end{tabular} \\
\hline 1997 & 1230 & 154 & 1338 & \begin{tabular}{|r|}
11.51 \\
\end{tabular} \\
\hline 1998 & 1028 & 124 & 1101 & 11.26 \\
\hline 1999 & 3211 & 106 & 3252 & 3.26 \\
\hline 2000 & 782 & 112 & 851 & 13.16 \\
\hline 2001 & 911 & 121 & 963 & 12.56 \\
\hline 2002 & 836 & 110 & 902 & 12.2 \\
\hline 2003 & 958 & 122 & 1027 & 11.88 \\
\hline 2004 & 912 & 110 & 967 & 11.38 \\
\hline 2005 & 948 & 137 & 1013 & 13.52 \\
\hline 2006 & 1051 & 165 & 1115 & 14.80 \\
\hline 2007 & 1015 & 161 & 1098 & 14.66 \\
\hline 2008 & 1010 & 127 & 1067 & 11.9 \\
\hline 2009 & 1027 & 133 & 1117 & 11.91 \\
\hline 2010 & 1091 & 164 & 1189 & 13.79 \\
\hline 2011 & 1030 & 147 & 1095 & 13.42 \\
\hline 2012 & 1248 & 171 & 1330 & 12.86 \\
\hline 2013 & 1106 & 176 & 1208 & 14.57 \\
\hline 2014 & 1010 & 150 & 1091 & 13.75 \\
\hline 2015 & 739 & 92 & 800 & 11.50 \\
\hline 2016 & 834 & 128 & 892 & 14.35 \\
\hline 2017 & 758 & 111 & 819 & 13.55 \\
\hline Total & 22735 & 2821 & 24235 & --- \\
\hline Media & 1082.619 & 134.3333 & 1154.0476 & 12.41 \\
\hline
\end{tabular}

Fuente: Elaboración propia con datos de INEGI (2020)

Por lo anterior, si bien se observa que la letalidad letalidad por atropellamiento peatonal. Sobre las por atropellamiento tiende a ser mayor por causas causas no atribuibles a los agentes, estas no son ajenas a acciones de los agentes. También, se pue- abordadas puntualmente en esta investigación, por de decir que cuando la causa se atribuye a acciones lo que futuras investigaciones podrían dirigirse a esdel peatón (cruzar o no cruzar la vialidad en inter- tudiarlas y analizarlas a mayor detalle en los modesección) la letalidad es porcentualmente mayor con los sociales y conductuales de CP y de mortalidad respecto a acciones atribuibles al conductor y que peatonal.

dicha letalidad es mayor en las no intersecciones más que en las intersecciones de las vialidades.

La evidencia estadística de la relación entre la mortalidad peatonal con las causas atribuibles junto con En consecuencia, la racionalidad acotada de cada el resto de las variables independientes y consideagente con respecto a la acción del otro, es decisiva rando la variable offset o de exposición a la mortalien cuanto a que son las acciones o causas atribui- dad se puede analizar a través de los resultados de bles al peatón las que presentan una mayor tasa de Modelos Poisson como se presenta a continuación. 
Tabla 4. Tasas de letalidad promedio (1997-2017) para variables de causa atribuible, lugar, horario, día, mes y alcaldía del accidente, en ZU de CdMx. (En negrillas valores máximos).

\begin{tabular}{|l|c|l|c|}
\hline \multicolumn{1}{|c|}{ Variable } & $\begin{array}{c}\text { Tasa de letalidad porcen- } \\
\text { tual considerando núme- } \\
\text { ro total de atropellados }\end{array}$ & Variable & $\begin{array}{c}\text { Tasa de letalidad porcentual } \\
\text { considerando número total } \\
\text { de atropellados }\end{array}$ \\
\hline Causa Conductor & 12.31 & Julio & 13.31 \\
\hline Causa Peatón & 14.35 & Agosto & 11.56 \\
\hline Otras Causas & $\mathbf{2 0 . 5 4}$ & Septiembre & 11.97 \\
\hline Accidente en intersección & 11.53 & Octubre & 12.28 \\
\hline Accidente en no & $\mathbf{1 3 . 1 9}$ & Noviembre & 14.02 \\
\hline intersección & $\mathbf{2 2 . 1 9}$ & Diciembre & 14.14 \\
\hline Madrugada & 12.63 & Álvaro Obregón & 18.08 \\
\hline Mañana & 8.91 & Azcapotzalco & 13.06 \\
\hline Tarde & 13.14 & Benito Juárez & 9.94 \\
\hline Noche & 12.49 & Coyoacán & 10.08 \\
\hline Lunes & 10.97 & Cuajimalpa de Morelos & 17.83 \\
\hline Martes & 11.79 & Cuauhtémoc & 9.47 \\
\hline Miércoles & 11 & Gustavo A. Madero & 16.73 \\
\hline Jueves & 11.97 & Iztacalco & 15.41 \\
\hline Viernes & 13.79 & Iztapalapa & 16.88 \\
\hline Sábado & $\mathbf{1 5 . 8 8}$ & Magdalena Contreras & 10.52 \\
\hline Domingo & 11.6 & Miguel Hidalgo & 9.41 \\
\hline Enero & 13.29 & Milpa Alta & 16.79 \\
\hline Febrero & 12.48 & Tláhuac & 14.41 \\
\hline Marzo & 11.75 & Tlalpan & 14.93 \\
\hline Abril & 13.99 & Venustiano Carranza & 13.6 \\
\hline Mayo & 13.24 & Xochimilco & $\mathbf{2 0 . 5 1}$ \\
\hline Junio & & \\
\hline Funn: & \\
\hline
\end{tabular}

Fuente: Elaboración propia con datos de INEGI (2020)

\section{Resultados de modelos Poisson}

El análisis de regresión del modelo Poisson simple indicó subdispersión a través del estadístico de Pearson (Pearson/GL =0.9002) (Tabla 5). Considerando lo anterior, el ajuste del Modelo PG permitió considerar dicha subdispersión estadísticamente, de esta forma los menores valores en el Criterio de Aikake $(\mathrm{AIC}=16779)$ y Bayesiano $(\mathrm{BIC}=17133)$ del Modelo PG demuestran mayor bondad de ajuste que el Poisson simple $(\mathrm{AIC}=16926.7674$ y $\mathrm{BIC}=$ 17264.0972). Igualmente el signo negativo del parámetro $\delta$ (delta $=-0.03572$ ) en el Modelo PG indica que el modelo considera la subdispersión de los datos. Además el Estadístico de la Razón de Verosimilitud (LRTEST= 151.7674 con un $P$-valor<0.0001) permite concluir que se rechaza significativamente la hipótesis nula de subdispersión en donde $\delta<0$, por lo que el Modelo PG permite abordar de manera correcta la subdispersión en los datos.

Con respecto a Colinealidad y siguiendo las indicaciones de Belsley (1991), para el modelo que consideró la variable año transformada como se indicó en metodología, se encontró un valor promedio del Factor de Inflación de Varianza de 1.94, el valor máximo de FIV encontrado fue de 4.25, por lo mismo con estos indicadores no hay evidencia de colinealidad en los datos, considerando posible existencia de colinealidad cuando se presentan FIV $\geq 10$. Se estimaron los índices de condición y sólo dos de ellos alcanzaron valores mayores a 10 y menores a 30 (11.39 y 24.67 respectivamente), lo cual es un posible indicador de colinealidad moderada. Empero 
Letalidad peatonal en Ciudad de México: análisis general desde una perspectiva de racionalidad limitada

Ramiro Flores-Xolocotzi

Hatso Hnini Revista de Investigación de Paisajes y Espacio Construido, Vol.1 Núm.1 Año 2021

no hubo dos o más variables bajo esos índices de variables son colineales. Por lo anterior, se concluye condición que alcanzaran simultáneamente valores que no existen problemas de colinealidad en la made proporción de varianza $\geq 0.5$, en el entendido que triz de variables independientes considerando la vaproporciones de varianza $\geq 0.5$ indicarían que esas riable año transformada.

Tabla 5. Resultados de Regresión (Modelo Poisson simple y Modelo PG).

\begin{tabular}{|c|c|c|c|c|c|c|}
\hline & \multicolumn{3}{|c|}{ Modelo Poisson } & \multicolumn{3}{|c|}{ Modelo PG } \\
\hline Variable & $\begin{array}{l}\text { Parámetro } \\
\text { estimado }\end{array}$ & $\mathrm{RR}$ & P-Valor & $\begin{array}{l}\text { Parámetro } \\
\text { estimado }\end{array}$ & $\mathrm{RR}$ & P-Valor \\
\hline Intercepto & -1.7453 & 0.1746 & $<0.0001$ & -1.7331 & 0.1767 & $<0.0001$ \\
\hline Lugar Intersección & -0.2341 & 0.7913 & $<0.0001$ & -0.2258 & 0.7979 & $<0.0001$ \\
\hline Causa Conductor & -0.1398 & 0.8695 & 0.0249 & -0.1292 & 0.8788 & 0.0321 \\
\hline Otras Causas & 0.5616 & 1.7535 & 0.0001 & 0.5619 & 1.754 & $<0.0001$ \\
\hline Mañana & -0.4749 & 0.6219 & $<0.0001$ & -0.4715 & 0.6241 & $<0.0001$ \\
\hline Tarde & -0.8669 & 0.4203 & $<0.0001$ & -0.864 & 0.4215 & $<0.0001$ \\
\hline Noche & -0.4025 & 0.6686 & $<0.0001$ & -0.3988 & 0.6711 & $<0.0001$ \\
\hline Martes & -0.1685 & 0.8449 & 0.0396 & -0.1669 & 0.8463 & 0.034 \\
\hline Miércoles & -0.0179 & 0.9823 & 0.8033 & -0.0164 & 0.9838 & 0.8116 \\
\hline \begin{tabular}{|l|} 
Jueves \\
\end{tabular} & -0.0649 & 0.9372 & 0.3643 & -0.0633 & 0.9387 & 0.3559 \\
\hline \begin{tabular}{|l|} 
Viernes \\
\end{tabular} & -0.0496 & 0.9516 & 0.4749 & -0.0478 & 0.9533 & 0.4727 \\
\hline Sábado & 0.138 & 1.148 & 0.0637 & 0.1401 & 1.1504 & 0.0503 \\
\hline Domingo & 0.1598 & 1.1733 & 0.0209 & 0.1617 & 1.1755 & 0.0149 \\
\hline Febrero & 0.1147 & 1.1215 & 0.2282 & 0.1157 & 1.1227 & 0.2035 \\
\hline Marzo & 0.1214 & 1.1291 & 0.1966 & 0.1224 & 1.1302 & 0.1733 \\
\hline Abril & 0.1359 & 1.1456 & 0.1615 & 0.1369 & 1.1467 & 0.1404 \\
\hline Mayo & 0.423 & 1.5265 & $<0.0001$ & 0.4241 & 1.5282 & $<0.0001$ \\
\hline \begin{tabular}{|l|} 
Junio \\
\end{tabular} & 0.343 & 1.4092 & 0.0004 & 0.344 & 1.4106 & 0.0002 \\
\hline \begin{tabular}{|l|} 
Julio \\
\end{tabular} & 0.3242 & 1.3829 & 0.0009 & 0.3252 & 1.3843 & 0.0005 \\
\hline Agosto & 0.1856 & 1.2039 & 0.0592 & 0.1866 & 1.2051 & 0.0485 \\
\hline Septiembre & 0.2704 & 1.3105 & 0.0043 & 0.2715 & 1.3119 & 0.0027 \\
\hline Octubre & 0.2476 & 1.2809 & 0.009 & 0.2486 & 1.2822 & 0.0062 \\
\hline Noviembre & 0.3604 & 1.4339 & 0.0001 & 0.3614 & 1.4353 & $<0.0001$ \\
\hline Diciembre & 0.4142 & 1.5132 & $<0.0001$ & 0.4153 & 1.5148 & $<0.0001$ \\
\hline Álvaro Obregón & -0.1222 & 0.885 & 0.3053 & -0.1214 & 0.8857 & 0.285 \\
\hline Benito Juárez & -0.4862 & 0.615 & 0.0003 & -0.4857 & 0.6153 & 0.0002 \\
\hline Coyoacán & -0.3895 & 0.6774 & 0.0027 & -0.3889 & 0.6778 & 0.0017 \\
\hline Cuajimalpa de Morelos & 0.3843 & 1.4686 & 0.0065 & 0.3847 & 1.4692 & 0.0046 \\
\hline Cuauhtémoc & -0.3519 & 0.7034 & 0.0013 & -0.3504 & 0.7044 & 0.0008 \\
\hline Gustavo A. Madero & 0.2744 & 1.3157 & 0.0089 & 0.2766 & 1.3186 & 0.0057 \\
\hline Iztacalco & 0.3217 & 1.3795 & 0.0132 & 0.3223 & 1.3803 & 0.0096 \\
\hline Iztapalapa & 0.1891 & 1.2082 & 0.0761 & 0.191 & 1.2105 & 0.0604 \\
\hline Magdalena Contreras & -0.4493 & 0.6381 & 0.0251 & -0.4492 & 0.6381 & 0.0197 \\
\hline Miguel Hidalgo & -0.1446 & 0.8654 & 0.248 & -0.144 & 0.8659 & 0.2252 \\
\hline Milpa Alta & 0.2268 & 1.2546 & 0.1422 & 0.2271 & 1.255 & 0.1264 \\
\hline Tláhuac & -0.0147 & 0.9854 & 0.9307 & -0.0145 & 0.9856 & 0.9293 \\
\hline Tlalpan & 0.0839 & 1.0875 & 0.4966 & 0.0845 & 1.0882 & 0.4722 \\
\hline Venustiano Carranza & 0.1453 & 1.1564 & 0.2004 & 0.1465 & 1.1578 & 0.1766 \\
\hline Xochimilco & 0.0538 & 1.0553 & 0.7467 & 0.054 & 1.0555 & 0.7299 \\
\hline Año & 0.3351 & 1.3981 & $<0.0001$ & 0.3414 & 1.4069 & $<0.0001$ \\
\hline
\end{tabular}


Letalidad peatonal en Ciudad de México: análisis general desde una perspectiva de racionalidad limitada

Ramiro Flores-Xolocotzi

Hatso Hnini Revista de Investigación de Paisajes y Espacio Construido, Vol.1 Núm.1 Año 2021

\begin{tabular}{|l|c|c|c|c|c|c|}
\hline Martes*Tarde & 0.1835 & 1.2014 & 0.1467 & 0.184 & 1.202 & 0.1293 \\
\hline Sábado*Noche & -0.3392 & 0.7123 & 0.0036 & -0.3387 & 0.7127 & 0.0025 \\
\hline Offset (LnPA) & 1 & 0 & - & 1 & 0 & - \\
\hline Parámetro $\delta$ (delta) & ----- & ----- & ----- & -0.03572 & & \\
\hline \multicolumn{7}{|c|}{ Criterios para valorar bondad de ajuste } \\
\hline \\
\hline \\
\hline
\end{tabular}

Elaboración propia con datos de INEGI (2020)

\section{Análisis del modelo Poisson Generalizado}

Considerando los resultados del Modelo PG y tomando en cuenta que el modelo se ajustó también con la variable offset (InPAi por CP) (Tabla 5), con los resultados de este modelo se analizará la prevalencia de fallecimientos considerando el total de atropellados por CP. Se hace la observación: que los signos de los parámetros obtenidos en el modelo de Poisson simple son idénticos a los obtenidos en el modelo generalizado, además de que su significancia es semejante. Por lo tanto el análisis que se hará con los resultados del modelo PG no se aparta de lo que se encuentra también en el Poisson Simple. De lo anterior, se observan diversos aspectos que se analizan a continuación.

Las variables de causa atribuible son estadísticamente significativas (Tabla 5). Se puede interpretar que la causa atribuible al conductor $(P$-valor $=0.032$, ver signo negativo del parámetro asociado) disminuye el logaritmo del conteo de muertes con respecto a causas atribuibles al peatón. Igualmente, si se toma el valor de RR para la misma causa, se observa que la mortalidad asociada a causa atribuible al conductor es $1-0.8788=0.1212$ (se puede multiplicar por 100 para expresarla en términos porcentuales). Esto es, cuando la causa de la CP es atribuible al conductor: la tasa de peatones atropellados fallecidos disminuye en $12.12 \%$ con respecto a la causa atribuible al peatón. Empero, para la variable Otras causas ( $P$ valor $<0.0001$ ), la tasa de peatones atropellados fallecidos se incrementa en $75 \%$ con respecto a la causa atribuible al peatón (obtenida de 1.7540$1=0.754)$.

Por lo anterior, hay causas ajenas a las acciones de los agentes que estadísticamente incrementan la tasa de peatones atropellados fallecidos con respec- to a causas atribuibles al peatón. Estas causas pueden ser fallas del vehículo y estar asociadas a las condiciones de este. Dichas causas al ser desconocidas por el peatón, constituyen un factor de incertidumbre e información incompleta para dicho agente considerando un modelo de racionalidad limitada dentro de los escenarios de las CP y su letalidad. Lo mismo podría suponerse para la condición de la vialidad (por ejemplo, presencia de baches) que el conductor pueda desconocer. Ambos supuestos, como se ha argumentado con anterioridad, forman parte del enfoque de la Racionalidad Limitada en los escenarios de colisiones peatonales y su impacto en el fallecimiento de los peatones.

A considerar también que la variable lugar del accidente (en intersección) es significativa $(P$-valor $<0.0001)$ con un signo negativo que indica que la $\mathrm{CP}$ en intersección disminuye el logaritmo del conteo de peatones fallecidos (ver Tabla 5). Además el valor de RR indica que en intersecciones la tasa de peatones atropellados fallecidos se reduciría en $20.21 \%$ con respecto a las CP que ocurren en las no intersecciones. Por lo anterior, considerando que de acuerdo al Reglamento de Tránsito de CdMx (2019), que establece que "la intersección" (también conocida como esquina) es "el nodo donde convergen dos o más vías, en la que se realizan movimientos direccionales del tránsito peatonal o vehicular de forma directa o canalizada por islas"; entonces de manera general "la no intersección" no es necesariamente un lugar de tránsito peatonal.

Consecuentemente, si el peatón decide cruzar la vía en las no intersecciones, estos lugares no necesariamente podrán contar con semáforos, pasos de cebra, incluso vibradores que forcen al vehículo a detenerse. Por lo mismo, la decisión del peatón de cruzar la vía en no intersecciones, estaría 
Letalidad peatonal en Ciudad de México: análisis general desde una perspectiva de racionalidad limitada

Ramiro Flores-Xolocotzi

Hatso Hnini Revista de Investigación de Paisajes y Espacio Construido, Vol.1 Núm.1 Año 2021

posiblemente relacionada con mayores tasas de mortalidad peatonal en CP. Habiendo un escenario así: existe un posible factor de incertidumbre e información incompleta tal como lo sugiere la Racionalidad Limitada para el conductor ante peatones que no crucen en las intersecciones o en sitios propicios para el cruce de vialidades.

Empero, será necesario que otras investigaciones analicen la frecuencia de ocurrencia de CP en "las intersecciones" y en "las no intersecciones", para analizar con mayor profundidad las hipótesis anteriores. Sin embargo, el análisis de las acciones de los agentes, podría reflejar el hábito de los peatones por cruzar mayormente en "las intersecciones". Es en "las intersecciones de las vialidades" en donde por Reglamento, se deben tener más facilidades para el cruce peatonal (presencia de semáforos, cebras y vibradores(reductores de velocidad), bolardos (impiden el paso de vehículos a zonas peatonales) y/o señales de velocidad máxima. Estas facilidades, en las intersecciones, tendrían como objetivo, reducir el número de colisiones peatonales e impactar en la disminución de las tasas de mortalidad peatonal. Consecuentemente es necesario que futuras investigaciones contemplen evaluar el impacto de dichas facilidades en el número de colisiones y en la mortalidad.

A lo anterior, hay que añadir la efectividad que puedan tener las infracciones a conductores o peatones que violen los reglamentos de tránsito, estos aspectos han sido abordados/mencionados por Rosén y Sander (2009); Schmidt-Daffy (2014); Shinar (2017); Tefft (2013) y Zhang et al. (2013). Los aspectos relacionados con atropellamientos y cumplimiento de los reglamentos de tránsito vehicular y desplazamientos peatonales, deberán ser considerados en futuros estudios peatonales y de movilidad urbana en Ciudad de México.

A las acciones e interacciones de los agentes en vialidades hay que considerar el resto de variables que pueden impactar en el número de fallecidos. En este modelo los horarios de accidentes (mañana, tarde y noche con categoría base de madrugada) son estadísticamente significativos (todos con un P-valor $<0.0001)$. En todos ellos, el signo negativo indica que de presentarse una de ellas: disminuye el logaritmo del conteo de fallecidos con respecto a la "madrugada". Esto significa que el conteo de peatones fallecidos tiende a ser mayor durante las madrugadas con respecto a los otros horarios. A destacar que la tasa de peatones atropellados fallecidos dis- minuye en mayor porcentaje en las tardes hasta en un $57.85 \%(1-0.4215)$ con respecto a las madrugadas.

Considerando una significancia estadística ( $P$-valor $<0.0001$ ), hay que destacar que en las noches, la tasa de fallecidos disminuye en $32.89 \%$ (1-0.6711); además se observa también una disminución en la tasa de $37.59 \%$ en las mañanas (1-0.6241). Estos dos resultados deben leerse con respecto a la categoría base de "madrugada". De esta forma, estos resultados son consistentes con el hecho de haber una mayor letalidad registrada en las madrugadas $(22.19 \%)$ en el período de tiempo estudiado para la CdMx, tal como se mencionó anteriormente. Para analizar estos resultados, hay que agregar que el flujo o volumen vehicular no se mantiene constante a lo largo del día, el cual es mayor durante el día y tarde que en las noches y madrugadas. Igualmente, las diferencias en las tasas estimadas de peatones fallecidos por horario, deben considerar que dichos horarios están asociados también a condiciones de luz natural/oscuridad y presencia de alumbrado público.

Con respecto a día de la semana, se observa que los martes son estadísticamente significativos ( $P$ valor $=0.034)$ y el signo del parámetro indica que el logaritmo del conteo de muertes es menor con respecto al lunes (base), por lo que la tasa de fallecidos disminuye con respecto a la base en un $15.37 \%$ (108463). Al contrario, para los sábados y domingos, las tasas de fallecidos se incrementan con respecto al lunes, en un $15 \%$ y $18 \%$ respectivamente. Se debe tomar en cuenta que los días pudieran estar asociados en menor o mayor grado con volúmenes de tráfico vehicular y movimiento de peatones considerando que los días entre semana son laborales y los fines de semana (sábado y domingo) tienden a ser días de asueto. Sin embargo, aunque el lunes sea un día laboral, las tasas de letalidad son mayores en los días de asueto. En consecuencia es necesario estudiar las diferencias en tráfico vehicular y los hábitos sociales de los agentes en días laborales y fines de semana.

Por todo lo anterior, existan contextos particulares de la conducta de los agentes que deben ser investigados con mayor detalle para explicar las diferencias en tasas de letalidad y número de fallecimientos. Por ejemplo, es necesario que futuras investigaciones consideren variables no incluidas en este trabajo, es el caso de variables como velocidad vehicular preferida por el conductor; contextos personales 
Letalidad peatonal en Ciudad de México: análisis general desde una perspectiva de racionalidad limitada

Ramiro Flores-Xolocotzi

Hatso Hnini Revista de Investigación de Paisajes y Espacio Construido, Vol.1 Núm.1 Año 2021

de los agentes incluyendo particularmente el consumo de alcohol/drogas (Shinar, 2017). También podría incluirse el tipo y número de vehículos involucrados en los atropellamientos y la disposición de servicios médicos en los lugares de atropellamiento. Además de incorporar en el análisis el aspecto legal del cumplimiento del Reglamento de Tránsito por parte de los agentes, tal como se aborda en otras investigaciones (Hebert y Porter, 2004).

En cuanto a meses, considerando los parámetros significativos todos con un nivel de significancia menor a 0.01 , de mayo a diciembre (a excepción de agosto con un $\mathrm{P}$-valor $=0.048$ ), todos estos meses tienen un signo positivo, el cual indica que los logaritmos de los conteos se incrementan con respecto a enero (Tabla 5). Con respecto a las tasas de peatones fallecidos, el menor incremento se presenta en agosto $(20 \%)$ y los mayores incrementos se presenta en mayo y diciembre con $52 \%$ y $51 \%$ respectivamente, ambas con respecto al mes base de enero.

Sobre las alcaldías (16 que dividen políticamente a CdMx), los parámetros significativos (al menos con un P-valor de 0.05) obtenidos para Magdalena Contreras, Benito Juárez, Coyoacán y Cuauhtémoc tienen un signo negativo que indica una disminución del logaritmo del conteo con respecto a la alcaldía base de Azcapotzalco. Teniendo la mayor disminución de la tasa de fallecidos con respecto a la base: la alcaldía Benito Juárez con $38.47 \%$. Sobre las alcaldías Cuajimalpa (P-valor $=0.0046)$, Gustavo A Madero (P-valor $=0.0057)$, Iztacalco $(P$-valor $=0.0096)$ e Iztapalapa (P-valor=0.06) todos con signos positivos, se observa el mayor incremento de tasa de fallecidos en Cuajimalpa con casi $47 \%$ respecto a la base.

Finalmente, la variable año es significativa ( $P$ valor $<0.0001$ ) con un signo positivo indicando que a medida que pasan los años, se incrementa la tasa de fallecidos.

Sobre las interacciones entre variables, la interacción sábado*noche es significativa $(P$ - valor $=0.0025)$ con un signo negativo, lo que indica que la tasa de peatones atropellados fallecidos disminuye en $28.79 \%$ con respecto a lunes y madrugadas.

Los resultados y análisis anteriores tienen que acotarse al hecho de que son ex post facto y no es un estudio dinámico interactivo. Por lo que los accidentes de tráfico junto con las colisiones peatonales en México podrían abordarse en futuras investigaciones a través de la teoría de juegos. Esta permitiría anali- zar las interacciones de conductores y peatones en intersecciones y no intersecciones mediante modelos como el juego de la coordinación, el juego del halcón y la paloma, juegos evolutivos y juegos dinámicos con suposiciones de información y racionalidad que se alejan de perspectivas clásicas y se acercan a las propuestas de RL (Elvik, 2014; Bjørnskau, 2017). La consideración de estos y más elementos permitirían también abordar la conducta de los agentes a través de la teoría prospectiva y prospectiva acumulada de Daniel Kahneman y Amos Tversky analizando diferentes contextos particulares de los agentes y sus decisiones, como lo han propuesto o realizado Chen et al. (2016); Shaoman y Ying (2015) y Schmidt-Daffy (2014).

\section{Conclusiones}

De los resultados y análisis anteriores se pueden concluir los aspectos que se señalan a continuación.

Se concluye que la letalidad por horario en promedio durante los años de estudio (1997 a 2017) es mayor en madrugadas $(22.19 \%)$, los días domingo $(15.88 \%)$, en el mes de diciembre $(14.14 \%)$ y en la alcaldía Xochimilco (20.51\%). Se encontró también que la letalidad por atropellamiento tiende a ser mayor por causas ajenas a acciones de los agentes (20.54\%). Estas causas ajenas deberán ser estudiadas puntualmente en otras investigaciones. Además, cuando la causa se atribuye a acciones del peatón la letalidad es porcentualmente mayor (14.35\%) con respecto a acciones atribuibles al conductor $(12.31 \%)$. Igualmente se tiene que considerar que la letalidad es mayor cuando la CP ocurre en las no intersecciones (13.19\%) más que en las intersecciones $(11.53 \%)$. Este resultado puede indicar el posible resultado de letalidad que se obtendría de la decisión del peatón, por cruzar o no cruzar en la intersección o esquina de la vialidad. Los resultados anteriores permiten concluir que la racionalidad acotada de cada agente con respecto a la acción del otro, puede ser decisiva en cuanto a que son las acciones o causas atribuibles al peatón más que las del conductor las que presentan una mayor tasa de letalidad por atropellamiento peatonal.

Sobre los resultados de las estimaciones de los modelos Poisson, se encontró lo siguiente:

1.- En cuanto a causas atribuibles, se concluye sobre letalidad, que el logaritmo del conteo de peatones fallecidos y la tasa de peatones atropellados (resultados del modelo PG) es significativamente mayor cuando el atropellamiento se atribuye a 
Letalidad peatonal en Ciudad de México: análisis general desde una perspectiva de racionalidad limitada

Ramiro Flores-Xolocotzi

Hatso Hnini Revista de Investigación de Paisajes y Espacio Construido, Vol.1 Núm.1 Año 2021

causas ajenas a la acción del conductor y del peatón. Con respecto a causas atribuibles a acciones de los agentes existe una mayor letalidad cuando el atropellamiento se atribuye al peatón que al conductor. Por lo anterior, hay causas ajenas a las acciones de los agentes y posiblemente desconocidas por ellos, que estadísticamente incrementan la tasa de peatones atropellados fallecidos. Estas condiciones ajenas posiblemente desconocidas por el peatón (condición y velocidad del vehículo), constituyen un factor de incertidumbre e información incompleta para dicho agente considerando un modelo de racionalidad limitada dentro de los escenarios de las CP y su letalidad. Lo mismo podría argumentarse para la condición de la vialidad que el conductor pueda desconocer.

2.- Igualmente se concluye sobre letalidad, que el logaritmo del conteo de peatones fallecidos y la tasa de peatones atropellados (resultados del modelo PG) es mayor cuando la CP ocurre en las no intersecciones más que en las intersecciones de las vialidades. Considerando que "las no intersecciones" pudieran ser lugares no propicios para cruzar la vialidad, esto pudiera incidir en una mayor letalidad y ser un factor de incertidumbre e información incompleta. Por lo anterior, considerando la racionalidad acotada de cada agente con respecto a la acción del otro, se puede concluir que las acciones atribuibles al peatón pueden provocar una mayor tasa de letalidad por atropellamiento peatonal.

3.- Sobre el resto de las variables que pueden impactar en el número de fallecidos se concluye que la tasa de letalidad disminuye en mayor porcentaje en las tardes con respecto a las madrugadas. Igualmente se encontró que el conteo de peatones fallecidos tiende a ser significativamente mayor durante las madrugadas con respecto a los horarios de mañana, tarde y noche. Con respecto a día de la semana, se concluye que en los martes el logaritmo del conteo es significativamente menor con respecto al lunes. Al contrario, para los sábados y domingos, las tasas de fallecidos se incrementan. Estos resultados deben analizarse considerando que los días entre semana son laborales y los fines de semana (sábado y domingo) tienden a ser días de asueto.

4.- Por todo lo anterior, existen contextos particulares de la conducta de los agentes que deben ser investigados en futuros trabajos para explicar las diferencias en tasas de letalidad y número de fallecimientos considerando también aspectos particulares del día y lugar en que ocurrió el atropellamiento.

\section{Referencias}

Allison, P. (2012). Logistic regression using SAS. Theory and application. USA, SAS Institute Inc. Bambarén, C. y Chú, M. (2013). Regulación del transporte y accidentes de tránsito por vehículos motorizados en el Perú. Revista Médica Herediana, 24(4):305-310.

Belsley, D. (1991). Conditioning diagnostics: Collinearity and weak data in regression. USA. Wiley \& Sons.

Bjørnskau, T. (2017). The Zebra Crossing Game. Using game theory to explain a discrepancy between road user behaviour and traffic rules. Safety Science, 92: 298-301. doi: 10.1016/ j.ssci.2015.10.007.

Chen, P., Wu, C. y Zhu, S. (2016). Interaction between vehicles and pedestrians at uncontrolled mid-block crosswalks. Safety Science, 82: 6876. doi: 10.1016/j.ssci.2015.09.016.

Chen, W., Zhuang, X., Cui, Z. y Ma, G. (2019). Drivers' recognition of pedestrian road-crossing intentions: Performance and process. Transportation Research Part F: Traffic Psychology and Behaviour, 64: 552-564. doi:10.1016/ j.trf.2019.07.004.

Chou, N. (2009). A flexible count data regression model using SAS® PROC NLMIXED. Paper 250-2009. SAS Global Forum 2009.

Consul, P. y Famoye, F. (1992). Generalized Poisson regression model. Communications in Statistics Theory and Methods, 21(1): 89-109. doi: 10.1080/03610929208830766.

Cui, Y., Kim, D. y Zhu, J. (2006). On the Generalized Poisson regression mixture model for mapping quantitative trait loci with count data. Genetics, 174(4): 2159-2172. doi: 10.1534/ genetics.106.061960.

Cummings, P. (2019). Analysis of Incidence Rates. USA. CRC Press (Taylor \& Francis Group).

Dong, C., Richards, S. H., Clarke, D. B., Zhou, X., y $\mathrm{Ma}, \mathrm{Z}$. (2014). Examining signalized intersection crash frequency using multivariate zeroinflated Poisson regression. Safety Science, 70: 63-69. doi: 10.1016/j.ssci.2014.05.006.

Eichelberger, A., McCartt, A. y Cicchino, J. (2018). Fatally injured pedestrians and bicyclists in the United States with high blood alcohol concentrations. Journal of Safety Research, 65: 1-9. doi: 10.1016/j.jsr.2018.02.004.

Elvik, R. (2014). A review of game-theoretic models of road user behaviour. Accident Analysis \& Prevention, 62: 388-396. doi: 10.1016/ j.aap.2013.06.016. 
Letalidad peatonal en Ciudad de México: análisis general desde una perspectiva de racionalidad limitada

Ramiro Flores-Xolocotzi

Hatso Hnini Revista de Investigación de Paisajes y Espacio Construido, Vol.1 Núm.1 Año 2021

Famoye, F., Wulu, J. y Singh, K. (2004). On the

Generalized Poisson regression model with an application to accident. Data. Journal of Data

Science, 2: 287-295. doi: 10.6339/

JDS.2004.02(3).167.

Flórez, C., Patiño, C., Rodríguez, J., Ariza, L. y González, R. (2018). Análisis multicausal de accidentes' de tránsito en dos ciudades de Colombia. Archivos de Medicina (Col), 18(1):1-20.

Fuller, R. (2005). Towards a general theory of driver behaviour. Accident Analysis \& Prevention, 37 (3): 461-472. doi: 10.1016/j.aap.2004.11.003.

Guimarães, A. y da Silva, A. (2019). Impact of regulations to control alcohol consumption by drivers: An assessment of reduction in fatal traffic accident numbers in the Federal District, Brazil. Accident Analysis \& Prevention, 127: 110-117. doi: 10.1016/j.aap.2019.01.017.

Harris, T., Yang, Z. y Hardin, J. (2012). Modeling underdispersed count data with generalized Poisson regression. The Stata Journal. 12(4): 736747. doi: 10.1177/1536867X1201200412.

Hebert, K. y Porter, B. (2004). The likelihood of becoming a pedestrian fatality and drivers' knowledge of pedestrian rights and responsibilities in the Commonwealth of Virginia. Transportation Research Part F: Traffic Psychology and Behaviour, 7(1): 43-58. doi: 10.1016/ j.trf.2003.11.001.

Hilbe, J. (2020). Modeling count data. USA. Cambridge University Press.

Husain, M. y Hossain, S. (2015). Modeling underdispersed count Data using Generalized Poisson regression approach. Global Journal of Quantitative Science, 2(4): 22-29.

INEGI. (2009). Síntesis metodológica de la estadística de accidentes de tránsito terrestre en zonas urbanas y suburbanas. México, INEGI.

INEGI. (2016). Síntesis metodológica de la estadística de accidentes de tránsito terrestre en zonas urbanas y suburbanas 2016. México, INEGI.

INEGI. (2020). Accidentes de tránsito terrestre en zonas urbanas y suburbanas. México, INEGI. Recuperado de https://www.inegi.org.mx/ programas/accidentes/.

Jiménez, D. (2010). Comportamiento peatonal. Tesis de Magister en Ciencias de la Ingeniería, mención Transporte. Chile. Universidad de Chile, Facultad de Ciencias Físicas y Matemáticas Departamento de Ingeniería Civil.

Lu, P. y Tolliver, D. (2016). Accident prediction model for public highway-rail grade crossings. Accident Analysis \& Prevention, 90: 73-81. doi: 10.1016/j.aap.2016.02.012.

Lukusa, M. y Kin, F. (2020) A Horvitz-type estimation on incomplete traffic accident data analyzed via a zero-inflated Poisson model. Accident Analysis and Prevention, 134: 1-9. doi: 10.1016/j.aap.2019.07.011.

Long, S. y Freese, J. (2014). Regression models for categorical variables using Stata. USA. Stata Press.

Másilková, M. (2017). Health and social consequences of road traffic accidents. Kontakt, 19(1): 43-47. doi: 10.1016/j.kontakt.2017.01.007.

Microsoft Excel (2021). Excel, versión 365. USA, Microsoft Corporation.

Mukherjee, D. y Mitra, S. (2019). A comparative study of safe and unsafe signalized intersections from the view point of pedestrian behavior and perception. Accident Analysis \& Prevention, 132:1-18. doi: 10.1016/j.aap.2019.06.010.

Plata, L., Mejía, I. y Accinelli, E. (2009). Sobre la teoría de decisiones bajo incertidumbre de VNM: antecedentes, extensiones y el papel de la racionalidad acotada de H. Simon. Revista mexicana de economía agrícola y de los recursos naturales, 2(3): 45-90.

Plata, L. (2013). Hay una mejor teoría para tomar decisiones bajo incertidumbre. Revista Mexicana de Economía y Finanzas, 8(1): 1-24.

Quistberg, D., Howard, E., Ebel, B., Moudon, A., Saelens, B., Hurvitz, P. y Rivara, F. (2015). Multilevel models for evaluating the risk of pedestrian-motor vehicle collisions at intersections and mid-blocks. Accident Analysis \& Prevention, 84: 99-111. doi: 10.1016/ j.aap.2015.08.013

Reglamento de Tránsito de CdMx (2019). Nuevo reglamento de tránsito Ciudad de México 2019. Gobierno de la Ciudad de México. México.

Rosén, E. y Sander, U. (2009). Pedestrian fatality risk as a function of car impact speed. Accident Analysis \& Prevention, 41(3), 536-542. doi: 10.1016/j.aap.2009.02.002.

Ruiz, J. y Herrera, A. (2016). Accidentes de tránsito con heridos en Colombia según fuentes de información: caracterización general y tipologías de accidentes. CES Psicología, 9(1):1-15.

SAS software. (2021). Statistical Analysis System Software, versión 9.4. NC, USA, SAS Institute Inc.

Secretaría de Salud. (2018). Informe sobre la Situación de la Seguridad Vial, México 2017. México, Secretaría de Salud. 


\section{Ramiro Flores-Xolocotzi}

Hatso Hnini Revista de Investigación de Paisajes y Espacio Construido, Vol.1 Núm.1 Año 2021

Schmidt-Daffy, M. (2014). Prospect balancing theory:

Bounded rationality of drivers' speed choice.

Accident Analysis \& Prevention, 63: 49-64.

doi: 10.1016/j.aap.2013.10.028

Shinar, D. (2017). Traffic safety and human behavior. United Kingdom. Emerald Publishing Limited.

Schilirò, D. (2018). Economic decisions and Simon's notion of Bounded Rationality. International Business Research, 11(7): 64-75. doi: ibr.v11n7p64.

Simon, H. (2019). Reason in human affairs. California, United States. Stanford University Press.

Sivak, M. (2002). How common sense fails us on the road: contribution of bounded rationality to the annual worldwide toll of one million traffic fatalities. Transportation Research Part F: Traffic Psychology and Behaviour, 5(4): 259-269. doi: 10.1016/s1369-8478(03)00003-2.

Tang, T., Huang, H. y Shang, H. (2015). Influences of the driver's bounded rationality on micro driving behavior, fuel consumption and emissions. Transportation Research Part D:

Transport and Environment, 41: 423-432. doi: 10.1016/j.trd.2015.10.016.

Tefft, B. (2013). Impact speed and a pedestrian's risk of severe injury or death. Accident Analysis \& Prevention, 50: 871-878. doi: 10.1016/ j.aap.2012.07.022.

Thompson, L., Rivara, F., Ayyagari, R. y Ebel, B. (2012). Impact of social and technological distraction on pedestrian crossing behaviour: an observational study. Injury Prevention, 19(4): 232-237. doi: 10.1136/injuryprev-2012040601.

Värnild A., Tillgren P. y Larm P. (2020) What types of injuries did seriously injured pedestrians and cyclists receive in a Swedish urban region in the time period 2003-2017 when Vision Zero was implemented? Public Health, 181:59-64. doi: 10.1016/j.puhe.2019.11.019.

Zhang, G., Yau, K. y Chen, G. (2013). Risk factors associated with traffic violations and accident severity in China. Accident Analysis \& Prevention, 59:18-25. doi: 0.1016/j.aap.2013.05.004. 